\title{
Approach for Uncertainty Propagation and Robust Design in CFD Using Sensitivity Derivatives
}

\author{
Michele M. Putko* \\ Perry A. Newman** \\ Arthur C. Taylor III *** \\ Lawrence L. Green**** \\ Department of Mechanical Engineering \\ old Dominion University \\ Norfolk, VA 23529 \\ Multidisciplinary Optimization Branch \\ NASA Langley Research Center \\ Hampton, VA 23681
}

Presented at the AIAA $15^{\text {th }}$ Computational Fluid Dynamics Conference June 11-14, 2001, Anaheim, CA

*LTC, US Army, PhD Candidate, Old Dominion University, Norfolk, VA 2352

**Senior Research Scientist, NASA Langley Research Center, Hampton VA 23681

***Associate Professor, Old Dominion University, Norfolk, VA 23529

****Research Scientist, NASA Langley Research Center, Hampton VA 23681,Senior Member AIAA, I.l.green@nasa.larc.gov 



\title{
APPROACH FOR UNCERTAINTY PROPAGATION AND ROBUST DESIGN IN CFD USING SENSITIVITY DERIVATIVES
}

\author{
Michele M. Putko* \\ Old Dominion University, Norfolk, VA 23529 \\ Perry. A. Newman ${ }^{\dagger}$ \\ NASA Langley Research Center, Hampton, VA 23681 \\ Arthur C. Taylor $\mathrm{III}^{+}$ \\ Old Dominion University, Norfolk VA 23529 \\ Lawrence. L. Green $\$$ \\ NASA Langley Research Center, Hampton, VA 23681
}

\begin{abstract}
$\underline{\text { Abstract }}$
This paper presents an implementation of the approximate statistical moment method for uncertainty propagation and robust optimization for a quasi I-D Euler CFD code. Given uncertainties in statistically independent, random, normally distributed input variables, a first-and second-order statistical moment matching procedure is performed to approximate the uncertainty in the CFD output. Efficient calculation of both first- and second-order sensitivity derivatives is required. In order to assess the validity of the approximations, the moments are compared with statistical moments generated through Monte Carlo simulations. The uncertainties in the CFD input variables are also incorporated into a robust optimization procedure. For this optimization, statistical moments involving firstorder sensitivity derivatives appear in the objective function and system constraints. Second-order sensitivity derivatives are used in a gradient-based search to successfully execule a robust optimization. The approximate methods used throughout the analyses are found to be valid when considering robustness about input parameter mean values.
\end{abstract}

\section{Nomenclature}

$\begin{array}{ll}\text { A } & \text { nozzle area } \\ \text { a } & \text { geometric shape parameter } \\ \text { b } & \text { geometric shape parameter } \\ \text { b } & \text { vector of independent input variables } \\ \text { F } & \text { vector of CFD output functions } \\ \text { g } & \text { vector of conventional optimization constraints } \\ \text { k } & \text { number of standard deviations } \\ \text { M } & \text { Mach number at nozzle inlet } \\ \text { M } & \text { vector of Mach number at each grid point }\end{array}$

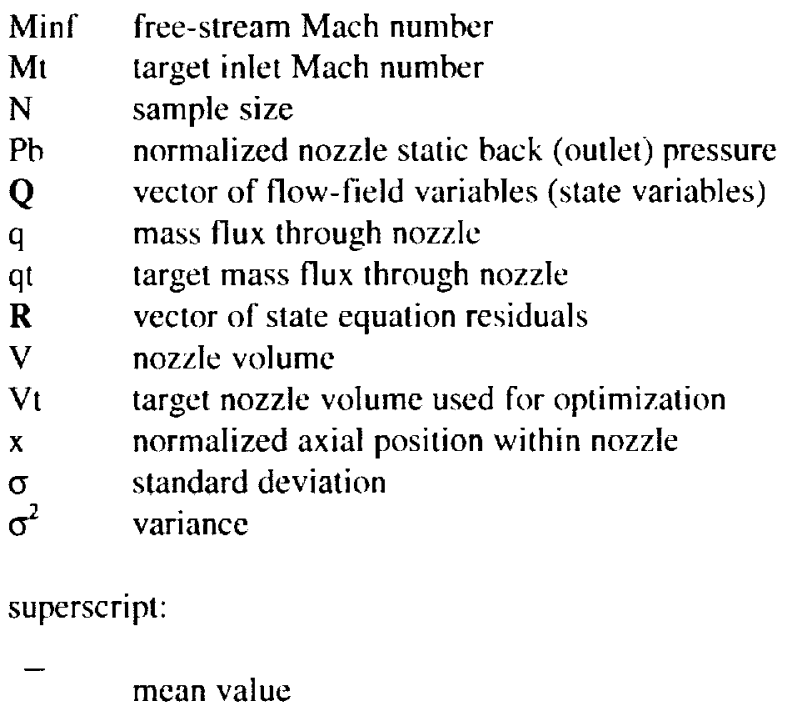

mean value

\footnotetext{
* LTC, US Army, Ph.D. Candidate, Department of Mechanical Engineering, mputko@tabdemo.larc.nasa.gov

tSenior Research Scientist, Multidisciplinary Optimization Branch, M/S 159, p.a.newman@larc.nasa.gov

Associate Professor, Department of Mechanical Engineering, ataylor@ions.odu.edu

$\$$ Research Scientist, Multidisciplinary Optimization Branch, M/S

159, AIAA senior member, I.I.green@larc.nasa.gov

This paper is declared a work of the U.S. Government and is not subject to copyright protection in the United States.
} 


\section{Introduction}

Gradient-based optimization of complex aerodynamic configurations and their components, utilizing high-fidelity Computational Fluid Dynamics (CFD) tools, continues as a very active area of research (see, for example, Ref. I, 2, and 3). In most of the CFD-based aerodynamic optimization and design studies to date, the input data and parameters have been assumed precisely known; we refer to this as deterministic or conventional optimization. When statistical uncertainties exist in the input data or parameters, however, these uncertainties affect the design and therefore must be accounted for in the optimization. Such optimizations under uncertainty have been studied and used in structural design disciplines (see, for example, Ref. 4, 5, 6, 7 and 8); we refer to these as non-deterministic or robust design optimization procedures.

Sensitivity derivatives (SD) of CFD code output, with respect to code input and parameters, contain information which can be used to direct the optimization search; that is, the objective and constraint gradients are functions of the CFD SD. Such SD can also be used to accurately approximate the CFD output in a small region, such as that near the mean value of a random variable. In Ref. 9, it is shown that a statistical First Order Second Moment (FOSM) method and Automatic Differentiation (AD) can be used to efficiently propagate input uncertainties through finite element analyses to approximate output uncertainty. This uncertainty propagation method is demonstrated herein for CFD code.

An integrated strategy for mitigating the effect of uncertainty in simulation-based design is presented in Ref. 10; this strategy consists of uncertainty quantification, uncertainty propagation, and robust design tasks or modules. Two approaches are discussed there for propagating uncertainty through sequential analysis codes: an extreme condition approach and a statistical approach. Both approaches can be efficiently implemented using SD. For CFD code, the former approach is demonstrated in Ref. 11 , whereas the latter approach is demonstrated herein using second moment approximations and SD. These uncertainty propagation methods have been developed and are being investigated as an alternative to propagation by direct Monte Carlo simulation for potentially expensive CFD analyses.

The present paper shows how the approximate statistical second moment methods, FOSM and the Second Order Second Moment (SOSM) counterpart, can be used in conjunction with SD to propagate input data uncertainties through CFD code to estimate output uncertainties. The FOSM approximation is then used to perform sample robust optimizations. For demonstration purposes, we assume that the input uncertainty quantification is given by independent normally-distributed random variables, and we demonstrate the strategy of Ref. 10 as applied to a CFD code module. This strategy is also applicable to correlated and/or non-normally distributed variables; however, the analysis and resulting equations become more complex.

The gradient-based robust optimization demonstrated herein requires second-order SD from the CFD code. A companion paper, Ref. 12, presents, discusses, and demonstrates the efficient calculation of second-order SD from CFD code using a method proposed, but not demonstrated, in Ref 13. This method, used herein, incorporates FO SD obtained by both forward-mode and reverse-mode differentiation in a non-iterative scheme to obtain SO SD.

To date, the only other demonstration or application of gradient-hased, robust optimization involving advanced or high-fidelity (nonlinear) CFD code that we have found was just recently presented in Ref. 14 and 15. The analytical statistical approximation of their objective function for robust optimization also required second-order SD. However, these studies employed a direct numerical random sampling technique to compute expected values at each optimization step in order to avoid the second-order SD. An example of linear aerodynamics involved in multidisciplinary performance optimization subject to uncertainty is found in Ref. 16.

Two other aspects need to be pointed out in regard to the robust optimization demonstrations for CFD code modules presented herein and also in Ref. 14 and 15. First, the sources of uncertainty considered were only those due to code input parameters involving geometry and/or flow conditions; i.e., due to sources external to the CFD code simulation. Other computational simulation uncertainties, such as those due to physical, mathematical and numerical modeling approximations (see Ref. 17 and 18) - essentially internal model error and uncertainty sources, were not considered. That is, the discrete CFD code analysis results were taken to be deterministically "certain" herein. Ultimately, all of these modeling sources of error and uncertainty must be assessed and considered. Sensitivity derivatives can also aid in this assessment (Ref. 19) since the adequacy of an internal model's (i.e., algorithm, turbulence, etc.) prediction capability generally depends, to some extent, on the modeling parameter values specified as input. 
Second, as discussed in Ref. 15, uncertainty classification with respect to an event's impact (from performance loss to catastrophic) and frequency (from everyday fluctuation to extremely rare) sets the problem formulation and solution procedure. Structural reliability techniques typically deal with risk assessment of infrequent but catastrophic failure modes, identifying the most probable point (MPP) of failure and its safety index. Here, we are addressing the assessment of everyday operational fluctuations on performance loss, not catastrophe. Consequently, we are most concerned with aero performance behavior due to probable fluctuations, i.e., near the mean of probability density functions (pdf). Structural reliability assessment is most concerned with improbable catastrophic events, i.e., probability in the tails of the pdf. Simultaneous consideration of both types of uncertainty is discussed in Ref. 16.

In Ref. 10 an integrated methodology for dealing with uncertainty in a simulation-based design is proposed and demonstrated for a linkage mechanism design. The integrated strategy of Ref. 10 for mitigating the effect of uncertainty includes (a) uncertainty quantification, (b) uncertainty propagation, and (c) robust design. The present study utilizes the strategy proposed in Ref. 10, hut differs in regard to uncertainty propagation and application. Here, we are considering the influence of uncertainty in CFD code input; that is, the effect of uncertainty in input geometry on aerodynamic shape-design optimization and the effect of uncertainty in flow conditions on design for flow control.

\section{$\underline{\text { Integrated Statistical Approach }}$}

Our implementation of the three aspects of the integrated strategy of Ref. 10 are as follows:

\section{Uncertainty Quantification}

In this study, we consider the influence of uncertainty in CFD input parameterization variables. We have assumed that these input variables are statistically independent, random, and normally distributed about a mean value. This assumption not only simplifies the resulting algebra and equations, but also serves to quantify input uncertainties. Furthermore, it is not an unreasonable assumption for input geometric variables subject to random manufacturing errors nor for input flow conditions subject to random fluctuations.

\section{Uncertainty Propagation}

Uncertainty propagation is accomplished by approximate statistical second moment methods
(FOSM and SOSM) where the required SD are obtained by hand or by AD (see Ref. 9 and 20). The first step in hoth FO and $\mathrm{SO}$ analyses is to approximate the CFD system output solutions of interest in Taylor series form. These approximations are formed to estimate the output value for small deviations of the input.

Given input random variables $\mathbf{b}=\left\{b_{1}, \ldots, b_{n}\right\}$ with mean $\overline{\mathbf{b}}=\left\{\overline{\mathrm{b}}_{1}, \ldots, \overline{\mathrm{b}}_{\mathrm{n}}\right\}$ and standard deviations, $\sigma_{b}=\left\{\sigma_{b_{1}}, \ldots, \sigma_{b_{n}}\right\}$, the CFD output function, $\mathbf{F}$, firstand second-order Taylor series approximations are given by

FO:

$$
\mathbf{F}(\mathbf{b})=\mathbf{F}(\overline{\mathbf{b}})+\sum_{i=1}^{n} \frac{\partial \mathbf{F}}{\partial b_{i}}\left(b_{i}-\overline{\mathbf{b}}_{i}\right)
$$

SO:

$$
\begin{aligned}
& \mathbf{F}(\mathbf{b})=\mathbf{F}(\overline{\mathbf{b}})+\sum_{i=1}^{n} \frac{\partial \mathbf{F}}{\partial b_{i}}\left(b_{i}-\bar{b}_{i}\right)+ \\
& +\frac{1}{2 !} \sum_{j=1}^{n} \sum_{i=1}^{n} \frac{\partial^{2} \mathbf{F}}{\partial b_{i} \partial b_{j}}\left(h_{i}-\bar{b}_{i}\right)\left(b_{j}-\bar{b}_{j}\right),
\end{aligned}
$$

where both first and second derivatives are evaluated at the mean values, $\overline{\mathbf{b}}$.

One then obtains expected values for the mean (first moment) and variance (second moment) of the output function, $\mathbf{F}$, which depend on the SD and input variances, $\sigma_{\mathbf{b}}{ }^{2}$. (Recall the variance is equivalent to the square of the standard deviation.) The mean of the output function, $\overline{\mathbf{F}}$, and standard deviation $\sigma_{\mathbf{F}}$, are approximated as

FO:

$$
\begin{aligned}
& \overline{\mathbf{F}}=\mathbf{F}(\overline{\mathbf{b}}) \\
& \sigma_{\mathbf{F}}{ }^{2}=\sum_{\mathrm{i}=1}^{\mathrm{n}}\left(\frac{\partial \mathbf{F}}{\partial \mathrm{b}_{\mathrm{i}}} \sigma_{\mathrm{b}_{1}}\right)^{2}
\end{aligned}
$$

SO:

$$
\begin{aligned}
& \overline{\mathbf{F}}=\mathbf{F}(\overline{\mathbf{b}})+\frac{1}{2 !} \sum_{j=1}^{n} \sum_{i=1}^{n} \frac{\partial^{2} \mathbf{F}}{\partial b_{i} \partial b_{j}} \sigma_{b_{1}} \sigma_{b_{j}} \\
& \sigma_{\mathbf{F}}{ }^{2}=\sum_{i=1}^{n}\left(\frac{\partial \mathbf{F}}{\partial b_{i}} \sigma_{b_{i}}\right)^{2}+\frac{1}{2 !} \sum_{j=1}^{n} \sum_{i=1}^{n}\left(\frac{\partial^{2} \mathbf{F}}{\partial b_{i} \partial b_{j}} \sigma_{b_{1}} \sigma_{b_{1}}\right)^{2} .
\end{aligned}
$$

where both first and second derivatives are evaluated at the mean values, $\overline{\mathbf{b}}$. Note in Eq. (4) that the secondorder mean output, $\overline{\mathbf{F}}$, is not at the mean values of input $\overline{\mathbf{b}}$, i.e., $\overline{\mathbf{F}} \neq \mathbf{F}(\overline{\mathbf{b}})$. 
Equations (3) represent a FO method and Eq. (4) a SO method for examining uncertainty propagation. The methods are straightforward with the difficulty largely lying in computation of the SD. The very efficient and effective method used here to obtain such derivatives is presented in a companion paper, Ref. 12.

\section{Robust Design}

Conventional optimization for an objective function, Obj, that is a function of the CFD output, $\mathbf{F}$, state variables, $\mathbf{Q}$, and input variables, $\mathbf{b}$, is expressed in $\mathrm{Eq}$. (5). Herein, the CFD state equation residuals, $\mathbf{R}$, are represented as an equality constraint, and other system constraints, g, are represented as inequality constraints. The input variables, $\mathbf{b}$, are precisely known, and all functions of $\mathbf{b}$ are therefore deterministic.

$\min \mathrm{Obj}$, subject to

$$
\operatorname{Obj}=\operatorname{Obj}(\mathbf{F}, \mathbf{Q}, \mathbf{b})
$$

$$
\begin{aligned}
& \mathbf{R}(\mathbf{Q}, \mathbf{b})=0 \\
& \mathbf{g}(\mathbf{F}, \mathbf{Q}, \mathbf{b}) \leq 0
\end{aligned}
$$

For robust design, the conventional optimization, Eq. (5), must be treated in a probabilistic manner. Given uncertainty in the input variables, $\mathbf{b}$, all functions in Eq. (5) are no longer deterministic. The design variables are now the mean values, $\overline{\mathbf{b}}=\left\{\overline{\mathrm{b}}_{1}, \ldots, \overrightarrow{\mathrm{b}}_{n}\right\}$, where all elements of $\overline{\mathbf{b}}$ are assumed statistically independent and normally distributed with standard deviations $\sigma_{b}$. The state equation residual equality constraint, $\mathbf{R}$, is deemed to be satisfied at the expected values of $\mathbf{Q}$ and $\mathbf{b}$, that is the mean values $\overline{\mathbf{Q}}$ and $\overline{\mathbf{b}}$ for the FO approximation. The objective function is cast in terms of expected values and becomes a function of $\overline{\mathbf{F}}$ and $\sigma_{\mathbf{r}}$. The other constraints are cast into a probabilistic statement: the probability that the constraints are satisfied is greater than or equal to a desired or specified probability, $P_{k}$. This probability statement is transformed (see Ref. 10) into a constraint involving mean values and standard deviations under the assumption that variables involved are normally distributed. The robust optimization can be expressed as

$$
\min \text { Obj, } \quad \operatorname{Obj}=\operatorname{Obj}\left(\overline{\mathbf{F}}, \sigma_{\mathbf{F}}, \overline{\mathbf{Q}}, \overline{\mathbf{b}}\right)
$$

subject to

$$
\begin{aligned}
& \mathbf{R}(\overline{\mathbf{Q}}, \overline{\mathbf{b}})=0 \\
& \mathbf{g}(\overline{\mathbf{F}}, \overline{\mathbf{Q}}, \overline{\mathbf{b}})+k \sigma_{\mathrm{g}} \leq 0,
\end{aligned}
$$

where $k$ is the number of standard deviations, $\sigma_{\mathrm{g}}$, that the constraint $\mathrm{g}$ must be displaced in order to achieve the desired or specified probability, $\mathrm{P}_{\mathrm{k}}$. For the FOSM approximation, standard deviations $\sigma_{\mathrm{F}}$ and $\sigma_{\mathrm{g}}$ are of the form given in Eq. (3) involving first-order $S D$.
Therefore, a gradient-based optimization will then require second-order $\mathrm{SD}$ to compute the objective and constraint gradients. Note that for the SOSM approximation, third-order SD would be required for these gradients.

The calculation of second-order SD for CFD code, such as those required for SOSM and robust optimization with FOSM, was demonstrated in Ref. 13; the efficient calculation used herein is demonstrated and discussed in a companion paper, Ref. 12. Both hand differentiation and AD via the ADIFOR tool (Ref. 21,22 and 23) werc used. Both conventional and robust optimizations were performed using the Sequential Quadratic Programming (SQP) method option in the Design Optimization Tools, DOT (Ref. 24).

\section{Application to Quasi 1-D Euler CFD}

A very simple example has been chosen to demonstrate the propagation of input uncertainty through CFD code and its effect on optimization. Two separate applications are presented; the first involving propagation of geometric uncertainties, the second involving propagation of flow parameter uncertainties. Both uncertainty analyses are performed with quasi one-dimensional Euler equations and boundary conditions describing subsonic flow through a variable area nozzle. The nozzle inlet is located at $x=0$ with area $A(x=0)=1$; the nozzle outlet is at $x=1$. The area distribution is given by

$A(x)=1-a x+b x^{2}$

The volume, $\mathrm{V}$, occupied by the nozzle, is the integration of $A(x)$ over the length $x=0$ to $x=1$ $\mathrm{V}=1-\frac{\mathrm{a}}{2}+\frac{\mathrm{b}}{3}$

where $\mathrm{a}$ and $\mathrm{b}$ are the input geometric parameters.

Three flow parameters are specified as input boundary conditions: the stagnation enthalpy, inlet entropy, and outlet static (back) pressure. The quasi 1-D Euler equation set is symbolically written as the state equation in Eq. (5); its residual, $\mathbf{R}$ is driven to (machine) zero for a solution.

For supersonic flow through a variable area nozzle, shock waves generally appear and the flow solution (objective, constraint, etc.) becomes noisy or nonsmooth (see Ref. 25 and the references cited therein). Care must be exercised with respect to obtaining and using the SD needed for gradient-based optimization (Ref. 25 and 26). Therefore, we chose to bypass issues related to this supersonic flow non-smoothness in these 
initial demonstrations of the present approach for uncertainty propagation and robust design for CFD code modules.

\section{Geometric Uncertainty Propagation}

For the discussion of geometric uncertainty propagation, geometric shape parameters a and $b$ will represent the statistically independent random input variables, $\mathbf{b}$. The Mach number distribution through the nozzle, $\mathbf{M}$, is viewed here as a component of the state variable, $\mathbf{Q}$; its value at the inlet, $M$, is the CFD output, F. Applying the approach previously outlined (recall Eq. (3) and (4)) yields the following first- and secondorder approximations of the output function, $\mathrm{M}$.

Input random variables: $\quad \mathbf{b}=\{\mathbf{a}, \mathbf{b}\}$

CFD output function: $\quad F=\{M\}$

FO Taylor series:

$M(a, b)=M(\bar{a}, \bar{b})+\frac{\partial M}{\partial a}(a-\bar{a})+\frac{\partial M}{\partial b}(b-\bar{b})$

SO Taylor series:

$$
\begin{aligned}
& M(a, b)=M(\bar{a}, \bar{b})+\frac{\partial M}{\partial a}(a-\bar{a})+\frac{\partial M}{\partial b}(b-\bar{b})+ \\
& +\frac{\partial^{2} M}{\partial a \partial b}(a-\bar{a})(b-\bar{b})+0.5\left(\frac{\partial^{2} M}{\partial a^{2}}(a-\bar{a})^{2}\right)+ \\
& +0.5\left(\frac{\partial^{2} M}{\partial b^{2}}(b-\bar{b})^{2}\right)
\end{aligned}
$$

The mean, $\bar{M}$, and standard deviation $\sigma_{M}$ of the output function are expressed as

FO:

$$
\begin{aligned}
& \bar{M}=M(\bar{a}, \bar{b}) \\
& \sigma_{M}{ }^{2}=\left(\frac{\partial M}{\partial a} \sigma_{a}\right)^{2}+\left(\frac{\partial M}{\partial b} \sigma_{b}\right)^{2}
\end{aligned}
$$

SO:

$$
\begin{aligned}
& \bar{M}=M(\bar{a}, \bar{b})+0.5\left(\frac{\partial^{2} M}{\partial a^{2}}\right) \sigma_{a}{ }^{2}+0.5\left(\frac{\partial^{2} M}{\partial b^{2}}\right) \sigma_{b}{ }^{2} \\
& \sigma_{M}{ }^{2}=\left(\frac{\partial M}{\partial a} \sigma_{a}\right)^{2}+\left(\frac{\partial M}{\partial b} \sigma_{b}\right)^{2}+0.5\left(\frac{\partial^{2} M}{\partial a^{2}} \sigma_{a}\right)^{2}+ \\
& +0.5\left(\frac{\partial^{2} M}{\partial b^{2}} \sigma_{b}{ }^{2}\right)^{2}+\left(\frac{\partial^{2} M}{\partial a \partial b} \sigma_{a} \sigma_{b}\right)^{2} .
\end{aligned}
$$

Predictions of $\mathrm{M}(\mathrm{a}, \mathrm{b}), \overline{\mathrm{M}}$, and $\sigma_{\mathrm{M}}$ for $\mathrm{FO}$ (Eq. (7) and (9)) and $\mathrm{SO}(\mathrm{Eq} .(8)$ and (10)) are compared with CFD solutions and Monte Carlo analyses based on CFD solutions, as given and discussed in the results section.

\section{Robust Shape Optimization}

Applying the conventional optimization previously described yields

$\min \mathrm{Obj}$

$$
\operatorname{Obj}=\operatorname{Obj}(M, a, b)
$$

$$
\begin{aligned}
& \mathbf{R}(\mathbf{M}, \mathbf{a}, \mathrm{h})=0 \\
& \mathrm{~V}(\mathbf{a}, \mathbf{b}) \leq 0,
\end{aligned}
$$

where the system constraint, $\mathrm{V}$, is a constraint on the nozzle volume and depends only on a and b; and our objective does not explicitly depend on $\mathbf{M}$.

Applying the robust optimization previously described yields

$\min$ Obj, $\quad \operatorname{Obj}=\operatorname{Obj}\left(\overline{\mathrm{M}}, \sigma_{M}, \overline{\mathrm{a}}, \overline{\mathrm{b}}\right)$

subject to

$$
\begin{aligned}
& \mathbf{R}(\overline{\mathbf{M}}, \overline{\mathbf{a}}, \overline{\mathrm{b}})=0 \\
& \mathrm{~V}(\overline{\mathrm{a}}, \overline{\mathrm{b}})+\mathrm{k} \sigma_{\mathrm{V}} \leq 0
\end{aligned}
$$

where

$\sigma_{\mathrm{V}}{ }^{2}=\left(\frac{\partial \mathrm{V}}{\partial \mathrm{a}} \sigma_{\mathrm{a}}\right)^{2}+\left(\frac{\partial \mathrm{V}}{\partial \mathrm{b}} \sigma_{\mathrm{b}}\right)^{2}$.

With a and b suhject to statistical uncertainties (which may be due to measurement, manufacturing, etc.), $\mathrm{V}$ becomes uncertain. Since $\mathrm{V}$ is linearly dependent on $a$ and $b$, it is also normally distributed. Therefore, its standard deviation, $\sigma_{V}$, is given exactly by Eq. (13).

To demonstrate the optimizations, a simple targetmatching problem is selected; a unique answer is obtained when an equality volume constraint is enforced. The CFD code is run for given a and $b$; the resulting $\mathrm{M}(\mathrm{a}, \mathrm{b})$ and corresponding $\mathrm{V}(\mathrm{a}, \mathrm{b})$ are taken as 
the target values $\mathrm{Mt}$ and $\mathrm{Vt}$, respectively. For this conventional optimization, the objective function and constraint function for $\mathrm{V}$ of $\mathrm{Eq}$. (11) become

$\operatorname{Obj}(M, a, b)=[M(a, b)-M t]^{2}$

$\mathrm{V}(\mathrm{a}, \mathrm{b})-\mathrm{Vt}=0$

enforced as

$\mathrm{V}(\mathrm{a}, \mathrm{b})-\mathrm{Vt} \leq 0$ and $\mathrm{Vt}-\mathrm{V}(\mathrm{a}, \mathrm{b}) \leq 0$

for the convenience of the optimizer.

For robust optimization using the FOSM approximation, the corresponding objective and constraints on V of Eq. (12) become

$\operatorname{Obj}\left(\overline{\mathrm{M}}, \sigma_{\mathrm{M}}, \overline{\mathrm{a}}, \overline{\mathrm{b}}\right)=[\overline{\mathrm{M}}(\overline{\mathrm{a}}, \overline{\mathrm{b}})-\mathrm{Mt}]^{2}+\sigma_{\mathrm{M}}{ }^{2}$

$\mathrm{V}(\overline{\mathrm{a}}, \overline{\mathrm{b}})-\mathrm{V} \mathrm{t}+\mathrm{k} \sigma_{\mathrm{V}}=0$

similarly enforced as

$\mathrm{V}(\overline{\mathbf{a}}, \overline{\mathbf{b}})-\mathrm{Vt}+\mathrm{k} \sigma_{\mathrm{V}} \leq 0$

and $V_{t}-V(\bar{a}, \bar{b})-k \sigma_{V} \leq 0$.

Note that for $\sigma_{a}=\sigma_{b}=0$ in Eq. (15), the conventional optimization is obtained. Also, in the probabilistic statement of the constraint on $\mathrm{V}$, it is assumed that the desired volume is less than or equal to Vt.

\section{Flow Parameter Uncertainty Propagation}

A second example of uncertainty in CFD involves fluctuations in input flow parameters. For the discussion of flow parameter uncertainty propagation, the free-stream Mach number, Minf, and the nozzle static back pressure, $\mathrm{Pb}$, will be taken as statistically independent random variables. Specifying the freestream Mach number sets the stagnation enthalpy. The Mach number distribution through the nozzle, $\mathbf{M}$, is again viewed as a component of the state variable, $\mathbf{Q}$; its value at the inlet, $\mathbf{M}$, is the $C F D$ output, $\mathbf{F}$. Applying the approach previously outlined yields equations which are similar to Eq. (7) through (10) but with

Input random variables: $\quad \mathbf{b}=\{\mathrm{Minf}, \mathrm{Pb}\}$

CFD output function: $\quad \mathbf{F}=\{\mathbf{M}\}$.

Again, predictions of $\mathrm{M}, \overline{\mathrm{M}}$, and $\sigma_{\mathrm{M}}$ for FO and $S O$ approximations are compared with CFD solutions and Monte Carlo analyses hased on CFD solutions, as given and discussed in the next section.

\section{Robust Design for Flow Control}

The conventional optimization is expressed as $\min$ Obj

subject to

$$
\mathrm{Obj}=\mathrm{Obj}(\mathrm{M}, \mathrm{Minf}, \mathrm{Pb})
$$

$$
\begin{aligned}
& \mathbf{R}(\mathbf{M}, \mathrm{Minf}, \mathrm{Pb})=0 \\
& \mathrm{q}(\mathrm{Minf}, \mathrm{Pb}) \leq 0,
\end{aligned}
$$

where $q$ is a constraint on the mass flux through the nozzle.

The robust optimization is expressed as

$$
\min \mathrm{Obj}, \quad \mathrm{Obj}=\mathrm{Obj}\left(\overline{\mathrm{M}}, \sigma_{\mathrm{M}}, \overline{\mathrm{M}} \mathrm{inf}, \overline{\mathrm{P}} \mathrm{b}\right)
$$

subject to

$$
\begin{aligned}
& \mathbf{R}(\overline{\mathbf{M}}, \overline{\mathrm{M}} \text { inf }, \overline{\mathrm{P}} b)=0 \\
& q(\overline{\mathrm{M}} \text { inf }, \overline{\mathrm{P}} \mathrm{b})+\mathrm{k} \sigma_{\mathrm{q}} \leq 0 .
\end{aligned}
$$

For the free stream Mach number, Minf, and the nozzle back pressure, $\mathrm{Pb}$, subject to statistical uncertainties, the mass flux, $\mathrm{q}$, becomes uncertain Since $\mathrm{q}$ is dependent on Minf and $\mathrm{Pb}$, its standard deviation, $\sigma_{4}$, may be approximated by

$$
\sigma_{\mathrm{q}}{ }^{2}=\left(\frac{\partial \mathrm{q}}{\partial \mathrm{Minf}} \sigma_{\mathrm{Minf}}\right)^{2}+\left(\frac{\partial \mathrm{q}}{\partial \mathrm{Pb}} \sigma_{\mathrm{Ph}}\right)^{2}
$$

Since $\mathrm{q}$ is not a linear function of Minf and $\mathrm{Pb}$, Eq. (18) is not exact (unlike the previous example where $\sigma_{v}$ was exactly known).

To demonstrate the optimizations, a simple targetmatching problem is again chosen. The CFD code is run for given $\mathrm{Minf}$ and $\mathrm{Pb}$; the resulting $\mathrm{M}$ and corresponding $q$ are taken as the target values $\mathrm{Mt}$ and $q \mathrm{q}$, respectively. For this conventional optimization, the objective function and constraint functions of Eq. (16) are

$\operatorname{Obj}(\mathrm{M}, \mathrm{Minf}, \mathrm{Pb})=[\mathrm{M}(\mathrm{Minf}, \mathrm{Pb})-\mathrm{Mt}]^{2}$

$\mathrm{q}(\mathrm{Minf}, \mathrm{Pb})-\mathrm{qt}=0$

enforced as

$\mathrm{q}(\mathrm{Minf}, \mathrm{Pb})-\mathrm{qt} \leq 0$ and $\mathrm{qt}-\mathrm{q}(\mathrm{Minf}, \mathrm{Pb}) \leq 0$.

For robust optimization using the FOSM approximation, the corresponding objective and constraint on $\mathrm{q}$ of $\mathrm{Eq}$. (17) can be shown as

$$
\begin{aligned}
& \mathrm{Obj}=\operatorname{Obj}\left(\overline{\mathrm{M}}, \sigma_{\mathrm{M}}, \overline{\mathrm{M}}_{\mathrm{inf}}, \overline{\mathrm{P}} \mathrm{b}\right) \\
& =[\overline{\mathrm{M}}(\overline{\mathrm{M}} \text { inf }, \overline{\mathrm{P}} \mathrm{b})-\mathrm{Mt}]^{2}+\sigma_{\mathrm{M}}{ }^{2} \\
& \mathrm{q}(\overline{\mathrm{M}} \text { inf, } \overline{\mathrm{P}} \mathrm{b})-\mathrm{q} t+\mathrm{k} \sigma_{\mathrm{q}}=0 \\
& \text { enforced as } \\
& \mathrm{q}(\overline{\mathrm{M}} \text { inf, } \overline{\mathrm{P}} \mathrm{b})-\mathrm{qt}+\mathrm{k} \sigma_{4} \leq 0
\end{aligned}
$$

Again note that for $\sigma_{\mathrm{Minf}}=\sigma_{\mathrm{pb}}=0$ in Eq. (20), the conventional optimization is obtained. Also, in the 
probabilistic statement of the constraint on $q$, it is assumed that the desired mass flux is less than or equal to ql.

\section{Sample Results \& Discussion}

Presentation and discussion of results for the sample quasi I-D Euler CFD problems are divided into four topics: function approximations, uncertainty propagation, pdf approximations, and robust optimization. For the first three topics, the approximations are assessed by comparison with direct CFD simulations.

\section{Function Approximations}

It is important to assess the Taylor series output function approximations with direct nonlinear CFD code simulations prior to presenting uncertainty propagation. If the CFD output function, $M$, is quasilinear with respect to the input variables of interest, one can expect first-order approximations to be reasonably good; that is, the FO moments given by Eq. (3) should match well with the moments produced by a Monte Carlo simulation. For a more nonlinear system, one naturally expects better accuracy with second order approximations; that is, uncertainty analyses which include SO terms should yield results which better predict the statistical moments produced by the Monte Carlo simulation.

Figures $\mathrm{I}-4$ show that for $\mathbf{F}=\mathbf{M}(\mathrm{a}, \mathrm{b}), \mathbf{M}$ behaves as a quasi-linear function in the neighborhood of $(\bar{a}, \bar{b})$, whereas for $\mathbf{F}=\mathbf{M}(\mathrm{Minf}, \mathrm{Pb}), \mathbf{M}$ is more nonlinear in the neighborhood of ( $\overline{\mathrm{M}} \mathrm{inf}, \overline{\mathrm{P}} \mathrm{b})$. In these figures, approximations of the CFD output functions, $M(a, b)$ and $\mathrm{M}(\mathrm{Minf}, \mathrm{Pb})$, using the first- (FO) and second-order (SO) Taylor series (as given in Eq. (7) and (8) for $\mathbf{M}(\mathrm{a}, \mathrm{b}))$, are compared to direct solution of the Euler CFD. In each example, two traces were considered through the design space. Trace 1 varied the first input variable, while the second remained fixed at its mean value, and vice versa for trace 2 . The required firstand second-order SD needed for construction of the FO and $\mathrm{SO}$ approximations were obtained by hand differentiation and $A D$ as discussed and presented in Ref. 12.

Nonlinear behavior of the CFD result is reasonably well approximated by the SO result in all plots; however, there does appear to be an inflection point in the CFD results given in Fig. 3. Note that the linear FO result is a good approximation in the geometric example; the flow parameter example is more nonlinear. At larger deviations from the mean, a linear approximation for $\mathrm{M}(\mathrm{Min} \Gamma, \mathrm{Pb})$ loses accuracy.

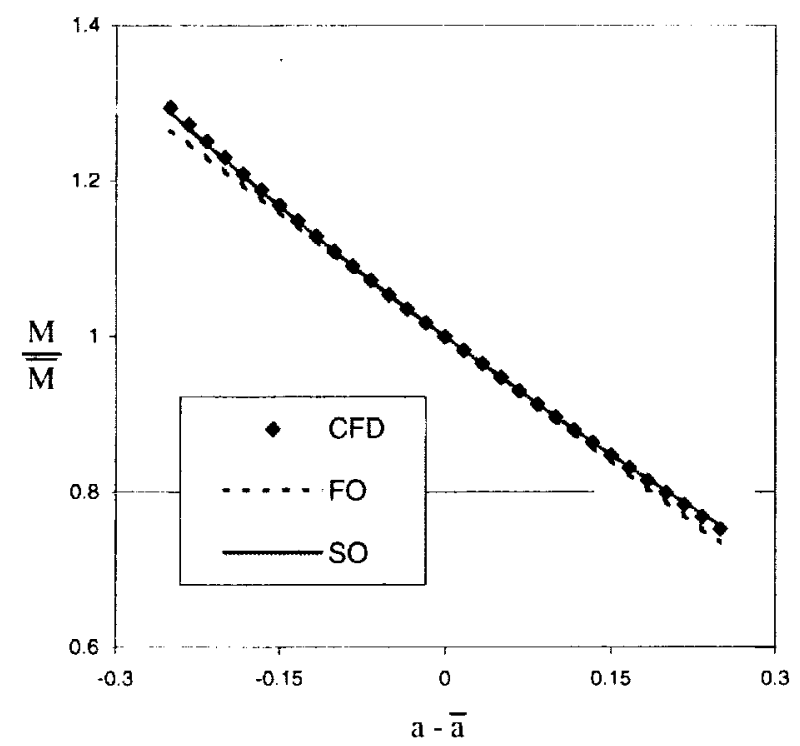

Fig. I. Comparison of Function Approximations vs. CFD Solution, Input Variable $b=\bar{b}$.

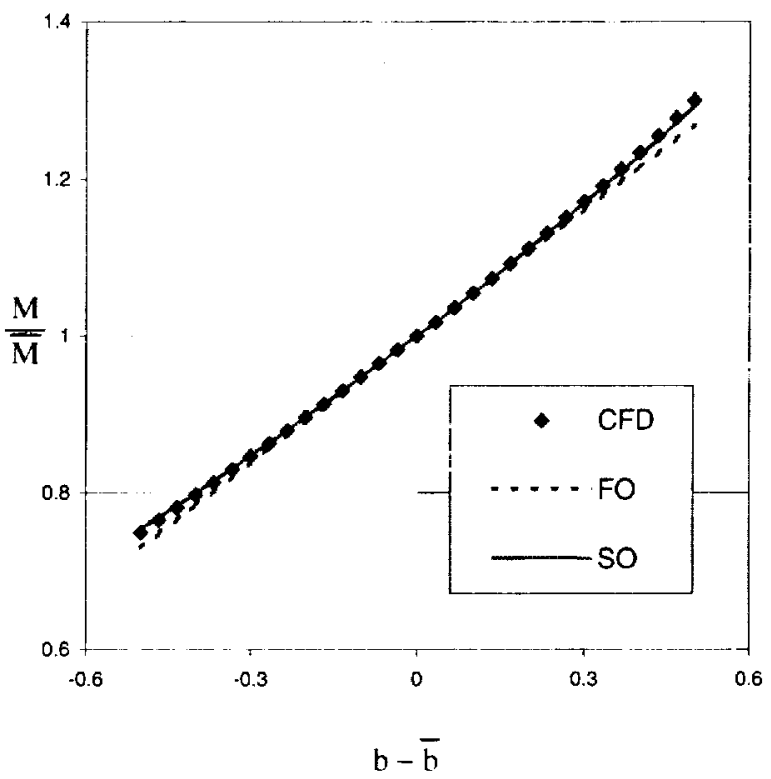

Fig. 2. Comparison of Function Approximations vs. CFD Solution, Input Variable $a=\bar{a}$. 


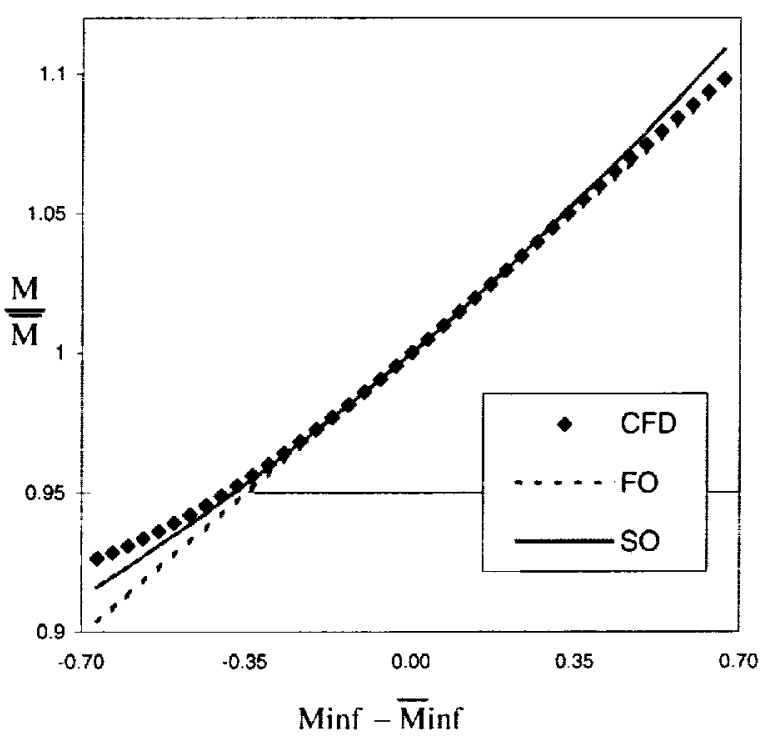

Fig. 3. Comparison of Function Approximations vs. CFD Solution, Input Variable $\mathrm{Pb}=\overline{\mathrm{P}} \mathrm{b}$.

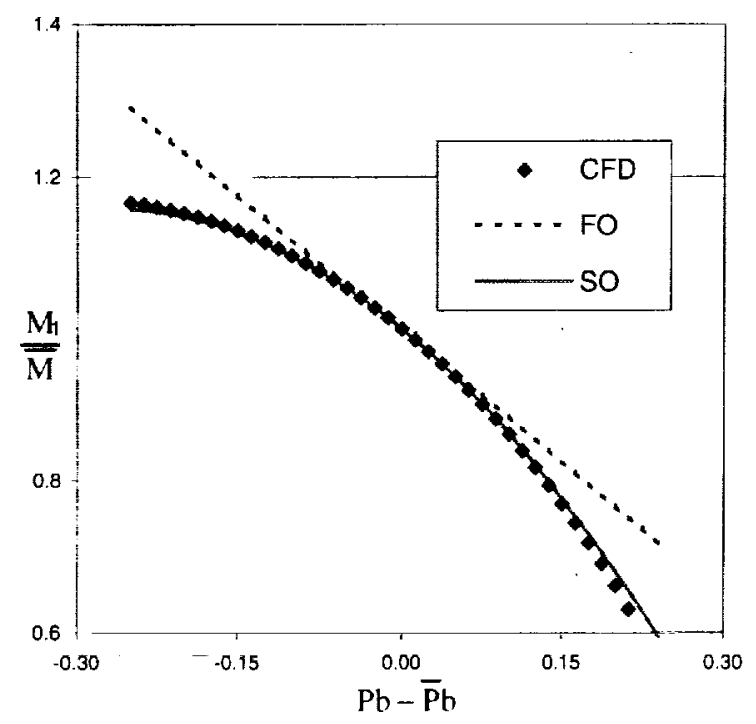

Fig. 4. Comparison of Function Approximations vs.

CFD Solution, Input Variable $\mathrm{Minf}=\overline{\mathrm{M}}$ inf .

\section{Uncertainty Propagation}

Approximation of the statistical first and second moments is done using equations Eq. (9) and (10) (geometric example), and corresponding equations for the flow parameter example. Again, both first- and second-order SD are required and the prediction is straightforward, given these derivatives. An independent verification of these approximate mean and standard deviation values is obtained here using direct Monte Carlo simulation with the quasi I-D Euler CFD code and standard statistical analyses of these Monte
Carlo results. The standard statistical analyses used were from MicroSoft (B) Excel 2000 and the random number generator MZRAN used was from Ref. 27. Tables 1 and 2 give results for the mean (first moment) and Tables 3 and 4 give results for the standard deviation (second moment) value comparisons. The input deviations $\left(\sigma_{\mathrm{a}}\right.$ and $\left.\sigma_{\mathrm{b}}\right)$ or $\left(\sigma_{\mathrm{Minf}}\right.$ and $\left.\sigma_{\mathrm{Ph}}\right)$, are taken to be equal and given in the second column of each table. The third column in each table gives the result from the Monte Carlo simulation, where the sample size (N) used was 3,000. The Monte Carlo error in its predicted mean is $\sigma_{M} / \sqrt{N}$, which is given in the fourth column of Tables $I$ and 2. The FO and SO approximate predictions are given in the last two columns of each table as percent difference from the Monte Carlo results.

Table 1. Percent Difference from Monte Carlo (MC) for $\mathrm{FO}$ and SO Predictions of $\overline{\mathrm{M}}(\overline{\mathrm{a}}, \overline{\mathrm{b}})$

\begin{tabular}{|c|c|c|c|c|c|}
\hline Case & $\begin{array}{c}\sigma_{\mathrm{a}}=\sigma_{\mathrm{b}} \\
\mathbf{M C}\end{array}$ & $\overline{\mathrm{M}}$ & $\begin{array}{c}\text { M Error } \\
\mathbf{M C}\end{array}$ & $\begin{array}{c}\text { F diff w/ MC Predict } \\
\text { FO diff w/ MC } \\
\text { SO Predict }\end{array}$ \\
\hline 1 & 0.01 & 0.4041 & 0.0187 & -0.0105 & 0.0656 \\
\hline 2 & 0.02 & 0.4040 & 0.0379 & 0.0716 & 0.1531 \\
\hline 3 & 0.04 & 0.4054 & 0.0756 & -0.2867 & 0.0383 \\
\hline 4 & 0.06 & 0.4055 & 0.1142 & -0.3012 & 0.4301 \\
\hline 5 & 0.08 & 0.4096 & 0.1557 & -1.3078 & -0.0209 \\
\hline
\end{tabular}

Table 2. Percent Difference from $\mathrm{MC}$ for $\mathrm{FO}$ and $\mathrm{SO}$ Predictions of $\overline{\mathrm{M}}(\overline{\mathrm{M}}$ inf, $\overline{\mathrm{P}} \mathrm{b})$

\begin{tabular}{|c|c|c|c|c|c|}
\hline Case & $\begin{array}{c}\text { Input } \sigma \\
\sigma_{\text {Mint }}=\sigma_{\mathrm{p}}\end{array}$ & $\begin{array}{l}\overline{\mathrm{M}} \\
\mathbf{M C} \\
\end{array}$ & $\begin{array}{c}\text { \% Error } \\
\text { MC }\end{array}$ & $\begin{array}{c}\text { \% diff w/ MC } \\
\text { FO Predict }\end{array}$ & $\begin{array}{l}\% \text { diff } w / M C \\
\text { SO Predict }\end{array}$ \\
\hline 1 & 0.01 & 0.3933 & 0.0056 & 0.0037 & -0.0269 \\
\hline 2 & 0.02 & 0.3932 & 0.0114 & 0.0187 & -0.1034 \\
\hline 3 & 0.04 & 0.3898 & 0.0229 & 0.8917 & 0.3991 \\
\hline 4 & 0.06 & 0.3889 & 0.0364 & 1.1251 & 0.0141 \\
\hline
\end{tabular}

Table 3. Percent Difference from MC for FO and SO Predictions of $\sigma_{\mathrm{M}}$. Geometric Example

\begin{tabular}{|c|c|c|c|c|}
\hline Case & $\begin{array}{c}\text { Input } \sigma \\
\sigma_{\mathrm{u}}=\sigma_{\mathrm{h}}\end{array}$ & $\begin{array}{c}\sigma_{\mathrm{M}} \\
\mathbf{M C}\end{array}$ & $\begin{array}{c}\text { \%dif } \mathbf{w} / \mathbf{M C} \\
\text { Fo Predict }\end{array}$ & $\begin{array}{c}\text { \%diff w/ MC } \\
\text { So Predict }\end{array}$ \\
\hline 1 & 0.01 & 0.0102 & -0.5773 & -0.5708 \\
\hline 2 & 0.02 & 0.0207 & -1.7026 & -1.6769 \\
\hline 3 & 0.04 & 0.0414 & -1.5794 & -1.4766 \\
\hline 4 & 0.06 & 0.0625 & -2.2590 & -2.0296 \\
\hline 5 & 0.08 & 0.0853 & -4.3987 & -4.0001 \\
\hline
\end{tabular}


Table 4. Percent Difference form MC for FO and SO Predictions of $\sigma_{\mathrm{M}}$. Flow Parameter Example

\begin{tabular}{|c|c|c|c|c|}
\hline Case & $\begin{array}{c}\text { Input } \sigma \\
\sigma_{\text {Minr }}=\sigma_{M^{\prime}}\end{array}$ & $\begin{array}{c}\sigma_{M} \\
\text { MC }\end{array}$ & $\begin{array}{c}\% \text { diff w/MC } \\
\text { Fo Predict }\end{array}$ & $\begin{array}{c}\% \text { diff w/ MC } \\
\text { So Predict }\end{array}$ \\
\hline 1 & 0.01 & 0.0030 & 1.1815 & 1.2473 \\
\hline 2 & 0.02 & 0.0062 & -1.5093 & -1.2533 \\
\hline 3 & 0.04 & 0.0125 & -4.1604 & -3.1680 \\
\hline 4 & 0.06 & 0.0199 & -4.4070 & -2.1938 \\
\hline
\end{tabular}

Note that mean and standard deviation approximations for output $\mathrm{M}$ agree well with the Monte Carlo results for small standard deviations in input variables, $\left(\sigma_{a}, \sigma_{\mathrm{b}}\right)$ or $\left(\sigma_{\mathrm{Minf}}, \sigma_{\mathrm{Pb}}\right)$, for both the $\mathrm{FO}$ and $\mathrm{SO}$ predictions. At higher standard deviations, the SO results tend to agree better with the Monte Carlo, especially in the flow parameter example. Table 3 illustrates the expected behavior, i.e., SO approximations are always better than FO and an increase in the standard deviation of input parameters monotonically increases the error associated with both predictions. Also note that the Monte Carlo simulation with a sample size of 3000 limits accuracy of the Monte Carlo results. This is apparent in the raggedness of the pdfs shown in the following section.

\section{Probability Density Function Approximations}

Given a mean and standard deviation of the CFD output function (from either a Monte Carlo simulation or an FO or SO prediction) and assuming a normal distribution, one may then construct a pdf to approximate the behavior of the non-deterministic output function. This approximation is compared to the pdf histogram generated from a Monte Carlo simulation in Figs. 5, 6, and 7. The hars depict the Monte Carlo histogram, and the solid curve is a normal distribution at the Monte Carlo mean value and Monte Carlo standard deviation as given in the previous tables. The Monte Carlo simulation size of 3000 is certainly not sufficient to obtain a smooth pdf. We note that both the FO and SO normal distributions are indistinguishable from this normal Monte Carlo curve at this scale. It is apparent that for either the quasi-linear functional dependence on a and b (Fig. 5), or for small input standard deviations of the flow parameters (Fig. 6), the statistical approximations are good for a significant region about the mean but tend to break down in predicting the tails of the distribution. This is significant, for if one is primarily interested in reliable failure predictions, as for structural design, this prediction may not be good enough. It is felt, however, that in aerodynamic performance optimization using CFD, where robustness about the mean is desired, these approximations may be good enough.

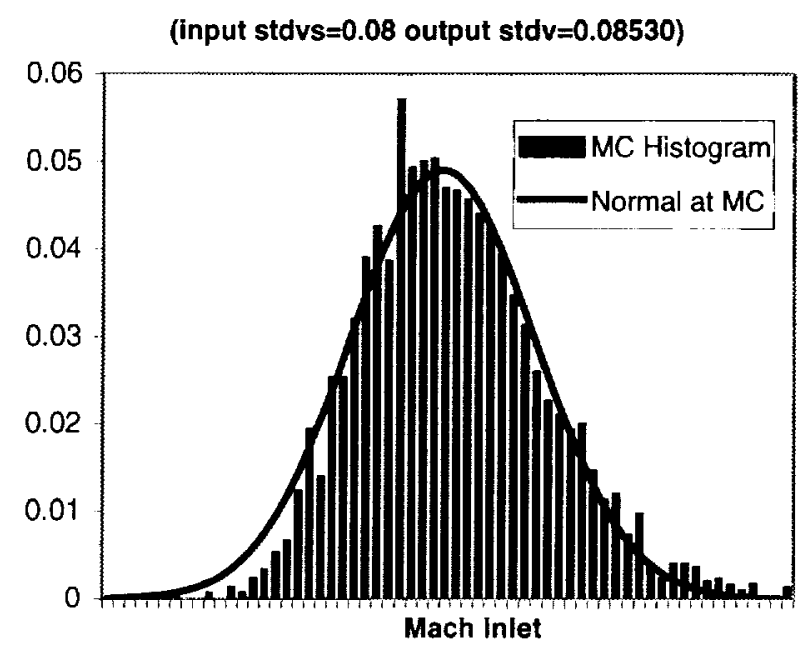

Fig. 5. Probability Density Function for $\mathrm{M}(\mathrm{a}, \mathrm{b})$ for $\sigma_{\mathrm{a}}=\sigma_{\mathrm{b}}=0.08$.

(input stdvs $=0.02$ output $s t d v=0.012276$ )

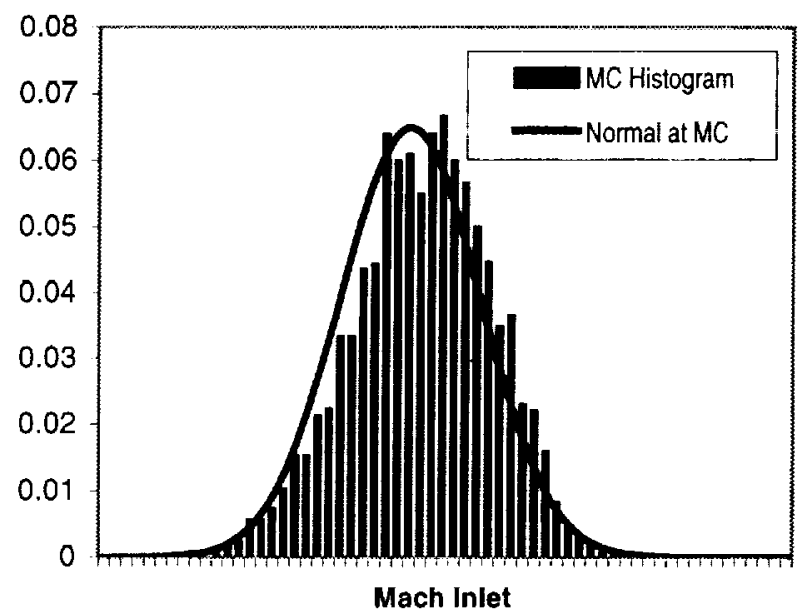

Fig. 6. Probability Density Function for M(Minf,Pb) for $\sigma_{\mathrm{Minf}}=\sigma_{\mathrm{Pb}}=0.02$.

It is not surprising that a nonlinear CFD output function behaves differently for randomness in different input variables. For $\mathrm{M}(\mathrm{Minf}, \mathrm{Pb})$ at higher input standard deviations $\left(\sigma_{\mathrm{Minf}}=\sigma_{\mathrm{Ph}}=0.06\right)$, the pdf of the output function is no longer normal. In Fig. 7 one can see the non-normal behavior of CFD output given normally distributed input variables Minf and $\mathrm{Pb}$. 
(input stdvs $=0.06$ output $s t d v=0.03795$ )

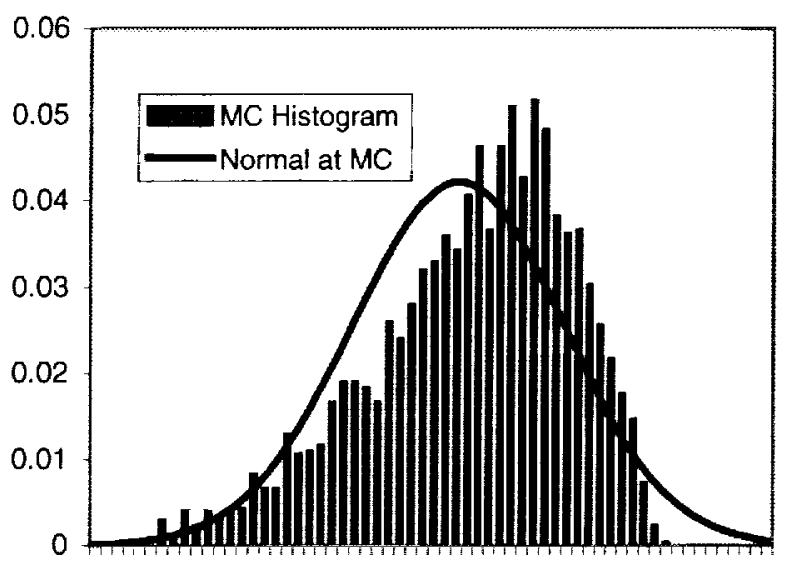

Mach Inlet

Fig. 7. Probability Density Function for M(Minf, $\mathrm{Pb})$ for $\sigma_{\text {Minf }}=\sigma_{\text {Ph }}=0.06$.

\section{Robust Optimizations}

Optimization results were generated using the quasi 1-D Euler CFD code and the procedure given by Eq. (5) and (6). As noted carlier, conventional optimization is obtained for $\sigma_{\mathrm{a}}=\sigma_{\mathrm{b}}=0$ or for $\sigma_{\text {Minf }}=\sigma_{\mathrm{Pb}}=0$. For the FOSM approximation, first-order SD are required to obtain $\sigma_{\mathrm{M}}$ and $\sigma_{\mathrm{V}}$ and $\sigma_{4}$; therefore, second-order SD will be required for their derivatives, which are used in the optimization. The second-order SD were obtained by the manner presented in Ref. 12, except for those associated with Eq. (18) where the first-order SD were finite differenced simply for convenience.

It is seen from Eq. (12) and (17) that the robust optimization results should depend on the probabilistic parameters $\left(\sigma_{\mathrm{a}}, \sigma_{\mathrm{b}}\right)$ or $\left(\sigma_{\mathrm{Mint}}, \sigma_{\mathrm{Pb}}\right)$, and $\mathrm{k}$. The desired probability, $P_{k}$, is that from the normal cumulative distribution function since $\sigma_{\mathrm{V}}$ and $\sigma_{\mathrm{q}}$ here are assumed to be normally distributed.

For each robust optimization example, two cases are presented. For case $1, P_{k}$ is fixed at $k=1$, i.e., $P_{1}=84.13 \%$, and the effect of increasing the input variable standard deviations is addressed. For case 2, the standard deviations of the input variables are fixed at 0.01 and $P_{k}$ increases.

Robust Shape Optimization Results- In Table 5, results for case 1 of the robust shape optimization are displayed. For $\sigma_{\mathrm{a}}=\sigma_{\mathrm{b}}$ ranging from 0 to 0.08 , optimal values for the input variables $(\bar{a}, \bar{b})$ are listed. As $\sigma_{\mathrm{a}}=\sigma_{\mathrm{b}}$ increases, so does $\sigma_{v}$. Accordingly, the mean values; $(\bar{a}, \bar{b})$, which minimize the objective function and satisfy the probabilistic constraint, become increasingly displaced from the target volume, $\mathrm{Vt}$. This is shown in Fig. 8. Mean values $(\bar{a}, \bar{b})$ change, keeping the mean value, $\bar{M}(\bar{a}, \bar{b})$, of the probabilistic output near the target value, Mt. The robust design points track the dashed curve for $\bar{M}=$ Mt with some displacement due to the $\sigma_{M}{ }^{2}$ term of the objective, Eq. (15). $V(\bar{a}, \bar{b})$ is displaced from the solid curve $V=V t$ by $k \sigma_{V}$, as required by the probabilistic constraint. This displacement can be viewed as the solution dependent or "effective" safety factor.

Table 5. Robust Shape Optimization Results with Increasing Input Parameter $\sigma$ for $\mathrm{k}=1$

\begin{tabular}{|c|c|c|c|c|c|c|}
\hline$\sigma_{\mathrm{d}}=\sigma_{\mathrm{h}}$ & $\overline{\mathrm{a}}$ & $\overline{\mathrm{b}}$ & $\mathrm{Obj}$ & $\overline{\mathrm{M}}$ & $\sigma_{\mathrm{M}}$ & $\sigma_{\mathrm{V}}$ \\
\hline 0.00 & 0.6001 & 0.3001 & 0.0000 & 0.4043 & 0.0000 & 0.0000 \\
\hline 0.02 & 0.6685 & 0.3667 & 0.0004 & 0.4036 & 0.0203 & 0.0120 \\
\hline 0.04 & 0.7338 & 0.4286 & 0.0016 & 0.4018 & 0.0406 & 0.0240 \\
\hline 0.06 & 0.7948 & 0.4841 & 0.0037 & 0.3984 & 0.0607 & 0.0360 \\
\hline 0.08 & 0.8534 & 0.5358 & 0.0065 & 0.3941 & 0.0804 & 0.0480 \\
\hline
\end{tabular}

In Fig. 9 the changing area distribution of the robust optimization is illustrated. As the standard deviations of design variables $(\bar{a}, \bar{b})$ increase, the optimal nondeterministic volume, $V(\bar{a}, \bar{b})$, significantly decreases.

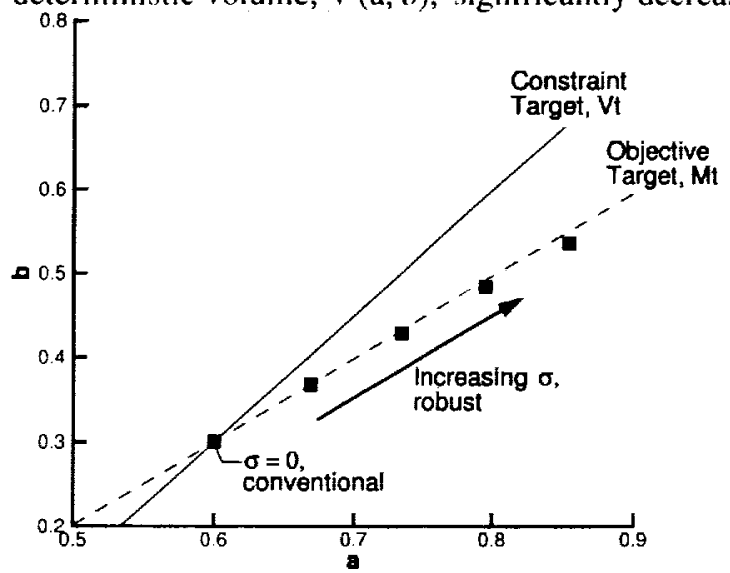

Fig. 8. Optimization results in design space $(a, b)$, $P_{k}$ fixed at $P_{1}$.

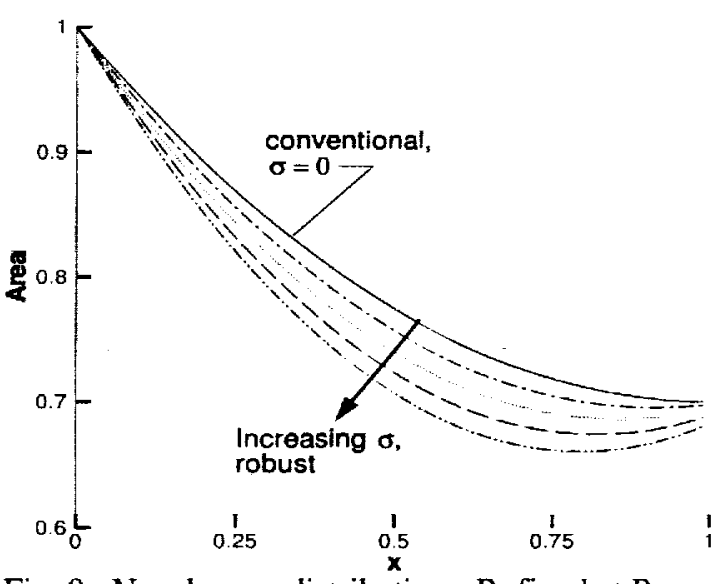

Fig. 9. Nozzle area distributions, $P_{k}$ fixed at $P_{k}$. 
The results for case 2 of the robust shape optimization, where $\sigma_{a}=\sigma_{b}$ is fixed at 0.01 , and $P_{k}$ increases from 50 percent to 99.99 percent ( $k=0$ to 4$)$ are given in Table 6 . Again mean values $(\overline{\mathbf{a}}, \bar{b})$ change, keeping the mean value, $\overline{\mathrm{M}}(\overline{\mathrm{a}}, \overline{\mathrm{b}})$, of the probabilistic output near the target value, $M t$. Since $\sigma_{a}=\sigma_{b}$ remains small, the $\sigma_{M}{ }^{2}$ term of the objective remains small, and the displacement of $\bar{M}$ from the dashed line depicting Mt due to the $\sigma_{M}{ }^{2}$ term remains small as shown in Fig. 10. With an increase in $P_{k}, V(\bar{a}, \bar{b})$ is displaced from the solid curve $V=V t$ by $k \sigma_{V}$, as required by the probabilistic constraint. Accordingly, the mean values, $(\bar{a}, \bar{b})$, which minimize the objective function and satisfy the constraint, again become increasingly displaced from those at the target volume, Vt. Note the significant displacement of the solution from the target volume when $P_{h}$ is large, i.e., when one is attempting to incorporate the tails of the pdf. In order to increase the probability of constraint satisfaction from 97.77 percent to 99.99 percent, one sees a significant change in $(\bar{a}, \bar{b})$ for a mere gain of 2 percent in constraint satisfaction.

Table 6. Robust Shape Optimization Results with Increasing $P_{k}$ for $\sigma=0.01$

\begin{tabular}{|c|c|c|c|c|c|c|c|}
\hline $\mathrm{K}$ & $\mathrm{P}_{\mathrm{k}}$ & $\overline{\mathrm{a}}$ & $\overline{\mathrm{b}}$ & $\mathrm{Obj}$ & $\overline{\mathrm{M}}$ & $\sigma_{\mathrm{M}}$ & $\sigma_{\mathrm{V}}$ \\
\hline 0 & 0.5000 & 0.5996 & 0.2995 & 0.000104 & 0.4041 & 0.0101 & 0.006 \\
\hline $\mathrm{I}$ & 0.841 .3 & 0.6246 & 0.3189 & 0.000118 & 0.4004 & 0.0101 & 0.006 \\
\hline 2 & 0.9772 & 0.6698 & 0.3687 & 0.000104 & 0.4041 & 0.0101 & 0.006 \\
\hline 3 & 0.9986 & 0.7052 & 0.4037 & 0.000104 & 0.4042 & 0.0102 & 0.006 \\
\hline 4 & 0.9999 & 0.7406 & 0.4388 & 0.000104 & 0.4043 & 0.0102 & 0.006 \\
\hline
\end{tabular}

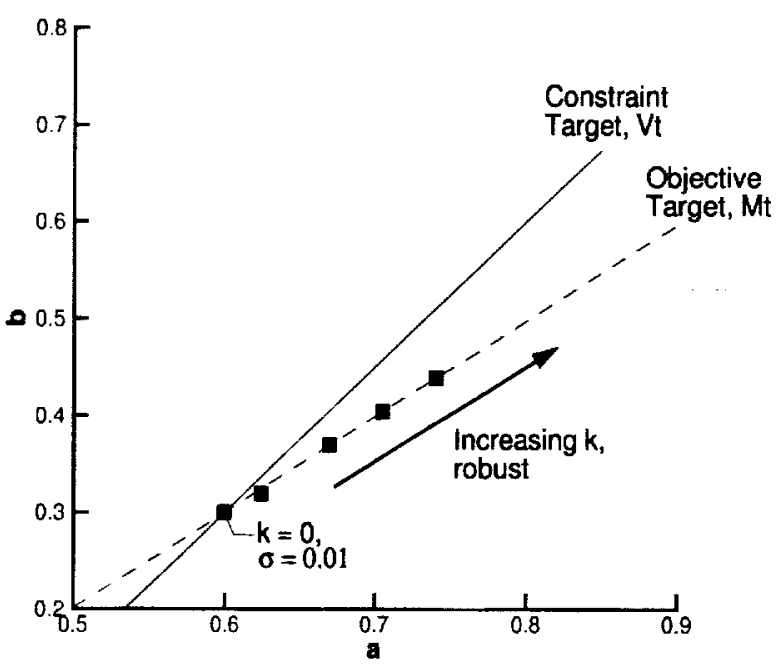

Fig. 10. Optimization results in design space $(a, b)$, $\sigma$ fixed at 0.01 .

Robust Design for Flow Control Results- Similar results are seen in the flow parameter example. In Table 7, the results for case 1 are displayed. For
$\sigma_{\mathrm{Minf}}=\sigma_{\mathrm{Pb}}$ ranging from 0 to 0.08 , optimal values for the input variables ( $\overline{\mathrm{M}}$ inf, $\overline{\mathrm{P}} \mathrm{b}$ ) are listed. As $\sigma_{\mathrm{Minf}}=\sigma_{\mathrm{Pb}}$ increases, so does $\sigma_{\mathrm{q}}$. Accordingly, the mean values, ( $\overline{\mathrm{M}}$ inf, $\overline{\mathrm{P}} \mathrm{b}$ ), which minimize the objective function and satisfy the constraint, become increasingly displaced from the target mass flux, qt. This is shown in Fig. 11. Mean values ( $\overline{\mathrm{M}}$ inf, $\overline{\mathrm{P}}$ ) change, keeping the mean value, $\overline{\mathbf{M}}(\overline{\mathrm{M}}$ inf, $\overline{\mathrm{P}} \mathrm{b})$, of the probabilistic output near the target value, $M t$. The robust design points track the dashed curve for $\bar{M}=M t$ with displacement due to the $\sigma_{M}{ }^{2}$ term of the objective, Eq. (17). The optimized mass flux, $q(\overline{\mathrm{M}}$ inf, $\overline{\mathrm{P}} \mathrm{h})$, is displaced from the solid curve $\mathrm{q}=\mathrm{qt}$ by $\mathrm{k} \sigma_{\mathrm{u}}$ as required by the probabilistic constraint.

Table 7. Robust Design for Flow Control Results with Increasing Input Parameter $\sigma$ for $k=1$

\begin{tabular}{|c|c|c|c|c|c|c|}
\hline$\sigma_{\operatorname{Min}}=\sigma_{\mathrm{Pb}}$ & $\overline{\mathrm{M}}$ inf & $\overline{\mathrm{Pb}}$ & $\mathrm{Obj}$ & $\overline{\mathrm{M}}$ & $\sigma_{\mathrm{M}}$ & $\sigma_{4}$ \\
\hline 0 & 0.3000 & 0.8000 & 0.0000 & 0.3933 & 0.0000 & 0.0000 \\
\hline 0.02 & 0.2861 & 0.7883 & 0.0001 & 0.3974 & 0.0116 & 0.0058 \\
\hline 0.04 & 0.2655 & 0.7801 & 0.0005 & 0.3985 & 0.0231 & 0.0112 \\
\hline 0.06 & 0.2555 & 0.7653 & 0.0012 & 0.4050 & 0.0327 & 0.0163 \\
\hline 0.08 & 0.2468 & 0.7498 & 0.0020 & 0.4118 & 0.0407 & 0.0209 \\
\hline
\end{tabular}

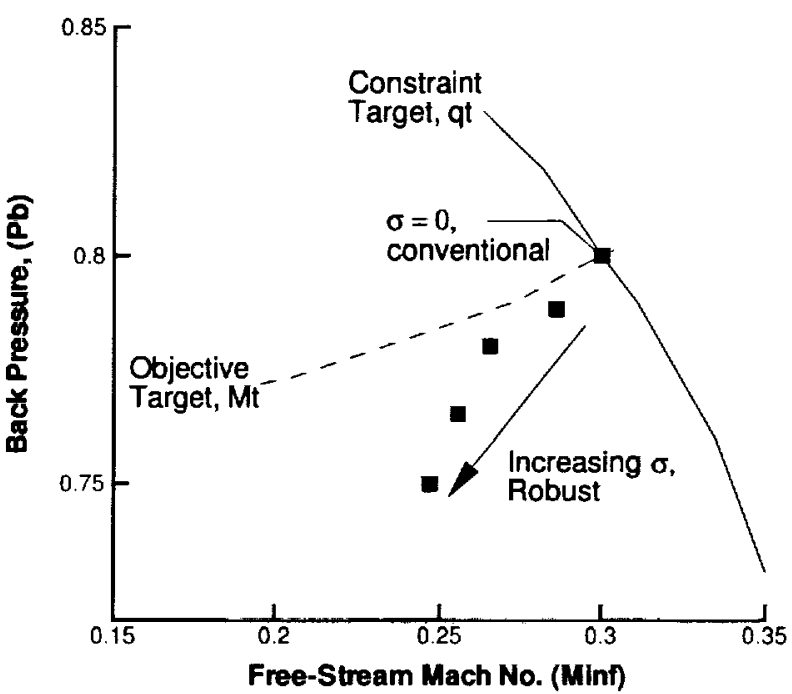

Fig. 11. Optimization results in design space (Minf, $\mathrm{Pb}$ ), $\mathrm{P}_{\mathrm{k}}$ fixed at $\mathrm{P}_{\mathrm{I}}$.

The results for case 2 of the robust design for flow control, where $\sigma_{\text {Minf }}=\sigma_{\mathrm{Pb}}$ is fixed at 0.01 , and $\mathrm{P}_{\mathrm{k}}$ increases from 50 percent to 99.99 percent, $(k=0$ to 4$)$ are given in Table 8. Again, mean values ( $\overline{\mathrm{M}}$ inf,$\overline{\mathrm{P}} \mathrm{b}$ ) change, keeping the mean value, $\overline{\mathbf{M}}(\overline{\mathrm{M}}$ inf,$\overline{\mathrm{P}} \mathrm{b})$, of the probabilistic output near the target value, Mt. As in the preceding example, since $\sigma_{\text {Minl }}=\sigma_{P b}$ remains small, the $\sigma_{M}{ }^{2}$ term of the objective remains small and the displacement due to the $\sigma_{M}{ }^{2}$ term remains small, as shown in Fig. 12. With an increase in 
$\mathrm{P}_{\mathrm{k}}, \mathrm{q}(\overline{\mathrm{M}}$ inf, $\overline{\mathrm{P}} \mathrm{b})$ is displaced from the solid curve $\mathrm{q}=\mathrm{qt}$ by $k \sigma_{4}$, as required by the probabilistic constraint. Accordingly, the mean values, ( $\overline{\mathrm{M}}$ inf, $\overline{\mathrm{P}} \mathrm{b}$ ), which minimize the objective function and satisfy the constraint again become increasingly displaced from the target mass flux, qt. Again, note the significant displacement from the target mass flux incurred in the higher probability optimizations, i.e., when one is attempting to incorporate the tails of the pdf.

Table 8. Robust Design for Flow Control Results with Increasing $P_{k}$ for $\sigma=0.01$

\begin{tabular}{|c|c|c|c|c|c|c|c|}
\hline$k$ & $\mathrm{P}_{\mathrm{k}}$ & $\overline{\mathrm{M}}$ inf & $\overline{\mathrm{P}} \mathrm{b}$ & $\mathrm{Obj}$ & $\overline{\mathrm{M}}$ & $\sigma_{\mathrm{M}}$ & $\sigma_{4}$ \\
\hline 0 & 0.5000 & 0.3000 & 0.8000 & 0.00003 & 0.3933 & 0.0060 & 0.0030 \\
\hline 1 & 0.8413 & 0.2919 & 0.7953 & 0.00003 & 0.3945 & 0.0059 & 0.0029 \\
\hline 2 & 0.9772 & 0.2825 & 0.7916 & 0.00003 & 0.3949 & 0.0059 & 0.0029 \\
\hline 3 & 0.9986 & 0.2688 & 0.7896 & 0.00003 & 0.3936 & 0.0060 & 0.0028 \\
\hline 4 & 0.9999 & 0.2598 & 0.7867 & 0.00003 & 0.3938 & 0.0060 & 0.0028 \\
\hline
\end{tabular}

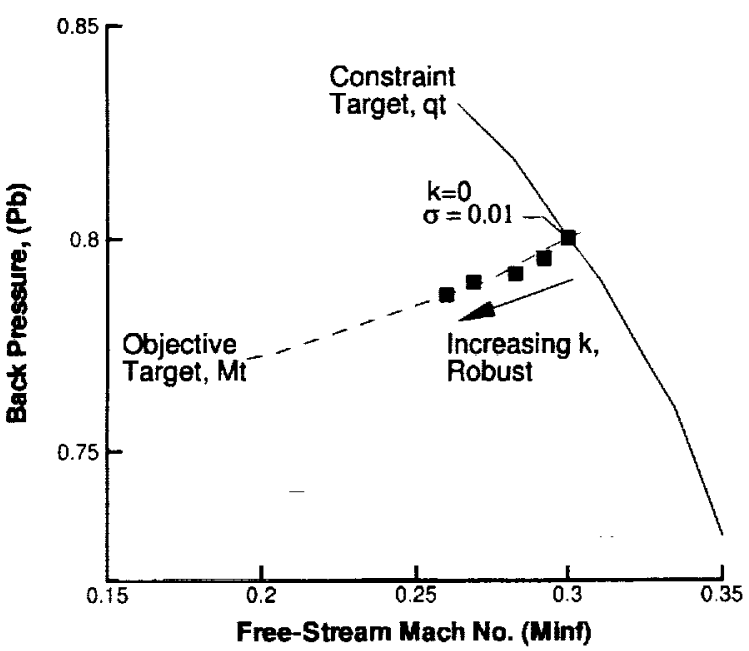

Fig. 12. Optimization results in design space (Minf,Pb), $\sigma$ fixed at 0.01 .

\section{Concluding Remarks and Challenges}

The present results represent an implementation of the approximate statistical moment method for uncertainty propagation and robust optimization for a quasi 1-D Euler CFD code. Assuming statistically independent, random, normally distributed input variables, a first- and second-order statistical moment matching procedure was performed to approximate the uncertainty in the CFD output. Efficient calculation of both first- and second-order sensitivity derivatives was employed and the validity of the approximations was assessed by comparison with statistical moments generated through Monte Carlo simulations. The uncertainties in the CFD input variables were incorporated into a robust optimization procedure where statistical moments involving first-order sensitivity derivatives appeared in the objective function and system constraints. Second-order sensitivity derivatives were used in a gradient-based robust optimization. The approximate methods used throughout the analyses were found to be valid when considering robustness about input parameter mean values.

Collectively, these results demonstrate the possibility for an approach to treat input parameter uncertainty and its propagation in gradient-based design optimization that is governed by complex CFD analysis solutions. It has been demonstrated on a very simple CFD code and problem; there are computational resource issues to be addressed in application to significant 2-D and 3-D CFD codes and problems. Some of these are addressed in the companion paper, Ref. 12. Work is presently in progress regarding application and demonstration using 2-D Euler CFD code.

\section{Acknowledgements}

The authors wish to thank Dr. Luc Huyse of the Institute for Computer Applications in Science and Engineering (ICASE) at NASA Langley for many helpful discussions regarding statistical aspects related to this study, and also Dr. Thomas Zang of the Multidisciplinary Optimization Branch at NASA Langley for his encouragement and support. The third author was partially supported by an ASEE Grant during the summer of 2000 at NASA Langley.

\section{References}

1. Newman, P. A., Hou; G. J.-W.; and Taylor III, A. C.: "Observations Regarding Use of Advanced CFD Analysis, Sensitivity Analysis, and Design Codes in MDO," in Alexandrov, N.M., and Hussaini, M.Y., Eds., Multidisciplinary Design Optimization: State of the Art, SIAM Proceedings Series, SIAM, Philadelphia, 1997., pp. 263-279; also ICASE Report 96-16, NASA CR 198293 (available electronically at www.icase.edu).

2. Newman, III, J. C.; Taylor III, A. C.; Barnwell, R. W.; Newman, P. A.; and Hou, G. J.-W.: "Overview of Sensitivity Analysis and Shape Optimization for Complex Aerodynamic Configurations," Journal of Aircraft. Vol. 36, No. 1, 1999, pp. 87-96. 
3. Jameson, A.; and Vassberg, J. C.: "Computational Fluid Dynamics for Aerodynamic Design: Its Current and Future Impact," AIAA-2001-05.38, Jan 2001.

4. Thanedar, P. B.; and Kodiyalam, S.: "Structural Optimization Using Probabilistic Constraints," Structural Optimization, Vol. 4, 1992, pp. 236-240 (also AIAA-91-0922-CP, 1991).

5. Parkinson, A.; Sorensen, C.; and Pourhassan, N.: "A General Approach for Robust Optimal Design," Jourmal of Mechanical Design. Vol. 115, No. 1, 1993, pp. 7480.

6. Chen, X.; Hasselman, T. K.; and Neill, D. J.: "Reliability Based Structural Design Optimization for Practical Applications," AIAA-97-1403, April 1997.

7. Du, X.; and Chen, W.: "Towards a Better Understanding of Modeling Feasibility Robustness in Engineering Design," American Sociely of Mechanical Engineers, Paper DAC-8565, Sept. 1999.

8. A Collection of Technical Papers, 40th AIAA/ASME/ASCE/AHS/ASC Structures, Structural Dynamics, and Materials Conference and Exhibit, AIAA Forum on Non-Deterministic Approaches, St. Louis, MO, April 12-15, 1999.

9. Chinchalkar, S.; and Taylor, D. L.: "Geometric Uncertainties in Finite Element Analysis," Computing Systems in Engineering, Vol. 5, No. 2, 1994, pp. 159170.

10. Du, X.; and Chen,W.: "Methodology for Managing the Effect of Uncertainty in Simulation-Based Design," AIAA J. , Vol. 38, No. 8, 2000, pp. 147/-1478.

11. Turgeon, E.; Pelletier. D.; and Borggaard, J.: "Sensitivity and Uncertainty Analysis for Variable Property Flows," AIAA 2001-0139, Jan. 2001.

12. Taylor III, A. C.; Green, L. L.; Newman, P. A.; and Putko, M. M.: "Some Advanced Concepts in Discrete Aerodynamic Sensitivity Analysis," AIAA-2001-2529.

13. Sherman, L.; Taylor III, A.; Green, L.; Newman, P.; Hou, G.; and Korivi, M.: "First- and Second-Order Aerodynamic Sensitivity Derivatives via Automatic Differentiation with Incremental Iterative Methods," Journal of Computational Physics, Vol. 129, No. 2, 1996, pp. 307-336.
14. Huyse, L.; and Lewis, R. M.: "Aerodynamic Shape Optimization of Two-dimensional Airfoils Under Uncertain Conditions," ICASE Report No. 2001-1, NASA CR-2001-210648, January 2001.

15. Huyse, L.: "Solving Problems of Optimization Under Uncertainty as Statistical Decision Problems." AIAA 2001-1519, April 2001.

16. Oakley, D. R.; Sues, R. H.; and Rhodes, G. S.: "Performance Optimization of Multidisciplinary Mechanical Systems Subject to Uncertainties, "Probabilistic Engineering Mechanics, Vol 13, No. 1, 1998, pp. 15-26.

17. Alvin, K. F.; Oberkampf, W. L.; Rutherford, B. M.; and Diegert, K. V.: "Methodology for Chacterizing Modeling and Discretization Uncertainties in Computational Simulation," Sandia Report SAND20005015, Mar 2000.

18. Oberkampf, W. L.; DeLand, S. M.; Rutherford, B. M.; Diegert, K. V.; and Alvin, K.F.: "A New Methodology for the Estimation of Total Uncertainty in Computational Simulation," AIAA-99-1612, April 1999 (See Ref 8., pp. 3061-308.3)

19. Green, L. L.; Newman. P. A.; and Haigler, K. J.: "Sensitivity Derivatives for Advanced CFD Algorithm and Viscous Modelling Parameters via Automatic Differentiation," Journal of Computational Physics, Vol. 125, No. 2, 1996, pp. 313-324 (also AIAA 933321 ).

20. Robinson, D. G.:"A Survey of Probabilistic Methods Used in Reliability, Risk and Uncertainty Analysis: Analytical Techniques I," Sandia Report SAND98-1189, June 1998.

21. Bischof, C. H.; Carle, A.; Corliss, G. F.; Griewank, A.; and Hovland, P.: "ADIFOR: Generating Derivative Codes from Fortran Programs," Scientific

Programming, Vol. 1, No. 1, 1992, pp. 1-29.

22. Bischof, C.; Carle, A.; Khademi, P.; and Mauer, A.: "Automatic Differentiation of FORTRAN," IEEE Computational Science \& Engineering, Fall 1996.

23. Carle, A.; and Fagan, M.: "Overview of Adifor 3.0," Department of Computational and Applied Mathematics, Rice University, CAAM-TR 00-02, Jan. 2000 . 
24. Anon., Design Optimization Tools - DOT Users

Manual: Version 4.20, Vanderplaats Research \&

Development, Inc., Colorado Springs, May 1995.

25. Dadone, A.; Valorani, M.; and Grossman, B.:

"Smoothed Sensitivity Equation Method for Fluid

Dynamic Design Problems," AIAA Journal, Vol. 38,

No. 3, 2000, pp. 418-426.

26. Zhang, X.; Pelletier, D.; Trepanier, J.; and

Camarero, R.: "Verification of Error Estimators for the Euler Equations," AIAA-2000-1001, January 2000.

27. Marsaglia, G.; and Zaman, A.: "Some Portable Very-Long-Period Random Number Generators,"

Computers in Physics, Vol.8, No.1, 1994, pp. 117-121. 